\title{
Electrophysiological measurements of spectral sensitivities: a review
}

R.D. DeVoe ${ }^{1}$,
J.M. de Souza ${ }^{2}$
and D.F. Ventura ${ }^{2}$

\author{
${ }^{1}$ School of Optometry, Indiana University, Bloomington, IN 47405, USA \\ ${ }^{2}$ Departamento de Psicologia Experimental, Instituto de Psicologia e \\ Centro de Neurociências e Comportamento, Universidade de São Paulo, \\ 05508-900 São Paulo, SP, Brasil
}

\author{
Correspondence \\ J.M. de Souza \\ Departamento de Psicologia \\ Experimental \\ Instituto de Psicologia, USP \\ Av. Prof. Mello Moraes, 1721 \\ 05508-900 São Paulo, SP \\ Brasil \\ Fax: 55 (011) 818-4357 \\ E-mail: johns@usp.br \\ Research supported by FAPESP \\ (No. 90/4883-0), FINEP \\ (No. 43.86.0779.00.01.02), and \\ CNPq-Aux. Int. (No. 523.303/95-5), \\ and by a Visiting Professor Grant \\ for R.D. DeVoe.
}

Received June 26, 1996

Accepted December 16, 1996

\begin{abstract}
Spectral sensitivities of visual systems are specified as the reciprocals of the intensities of light (quantum fluxes) needed at each wavelength to elicit the same criterion amplitude of responses. This review primarily considers the methods that have been developed for electrophysiological determinations of criterion amplitudes of slow-wave responses from single retinal cells. Traditional flash methods can require tedious dark adaptations and may yield erroneous spectral sensitivity curves which are not seen in such modifications as ramp methods. Linear response methods involve interferometry, while constant response methods involve manual or automatic adjustments of continuous illumination to keep response amplitudes constant during spectral scans. In DC or AC computerized constant response methods, feedback to determine intensities at each wavelength is derived from the response amplitudes themselves. Although all but traditional flash methods have greater or lesser abilities to provide on-line determinations of spectral sensitivities, computerized constant response methods are the most satisfactory due to flexibility, speed and maintenance of a constant adaptation level.
\end{abstract}

\section{Introduction}

The physiological study of color vision necessarily requires the determination of spectral sensitivity functions $S(\lambda)$ of cells of the visual system, beginning with photoreceptors. In turn, the spectral sensitivity of a photoreceptor depends on the absorption spectrum of its photopigment. According to the Principle of Univariance (1), “...every quantum that is effectively absorbed makes an equal contribution towards vision", independent of the wavelength of the absorbed
Key words

- Spectral sensitivity

- Color vision

- Electrophysiology

- Experimental automation

\footnotetext{
${ }^{1}$ Based on an old tradition in psychophysics, electrophysiological measurements of spectral sensitivities of human subjects are sometimes given in terms of the radiant energies instead of the quantum fluxes needed to elicit equal magnitudes of response.
}

light. This is not to say that all wavelengths are equally absorbed; they most assuredly are not. Therefore, the determination of a spectral sensitivity function involves determining the number of quanta per second at each wavelength that must fall upon a photoreceptor (how intense a light must be at each wavelength) to evoke an equal contribution towards vision ${ }^{1}$. The spectral sensitivities are then the reciprocals of these intensities. Such a spectral sensitivity curve is schematized in the bottom panel of Figure 1.

In electrophysiological studies, different 

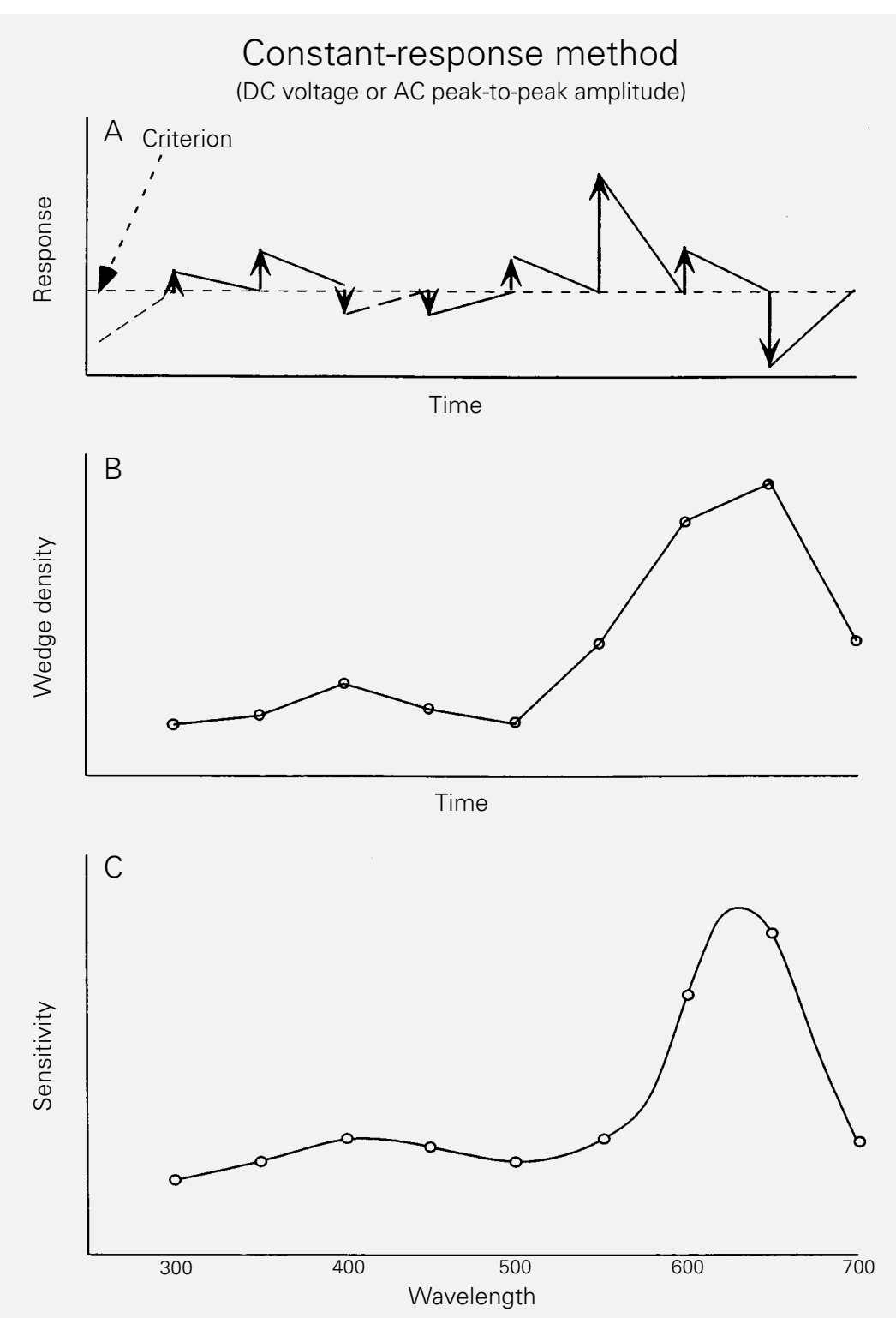

Figure 1 - Schematic illustration of constant-response methods. A shows a schematic response of the photoreceptor to wavelength and intensity changes. At the beginning, the wavelength is set to $300 \mathrm{~nm}$, and the wedge density is stepped down until the response reaches the criterion amplitude. Then the wavelength is stepped up to the next value, the response departs plus/minus from the criterion amplitude, and the wedge density is stepped in the appropriate direction until the response again reaches the criterion amplitude. This process continues until all wavelengths have been tested. $B$ illustrates the wedge density changes with time that correspond to the response changes in $A$. The quantum fluxes necessary to elicit criterion responses at each wavelength are calculated from the wedge densities in $B$, and the spectral sensitivity in $C$ is calculated as the reciprocal of these quantum fluxes. wavelengths are considered to make an equal contribution towards vision when they elicit the same magnitude of electrical response. For the most part, the electrical responses considered in this review will be graded receptor (non-spiking) potentials recorded intracellularly from photoreceptors and other visual neurons, but optic nerve discharges and electroretinograms (ERGs) will also be considered.

\section{Traditional flash methods}

Traditionally, $S(\lambda)$ functions of visual neurons have been measured by flash methods, in which monochromatic light pulses are presented to the dark-adapted eye while responses are recorded by means of an electrode. At their simplest, as schematized in Figure 2A, log intensities are adjusted up or down at each of a number of wavelengths until the response amplitudes equal some chosen criterion amplitude. This procedure is not often used, since it can be difficult to accurately judge amplitudes of response online and it can be tedious and time consuming to find just the right intensity at each wavelength. Instead, a more usual approach involves measuring curves of response amplitudes versus the logarithm of the intensity (V/logI functions) at different wavelengths across the spectrum $(2,3)$ and calculating $S(\lambda)$ in terms of the intensity required for some criterion response amplitude at each wavelength. This is schematized in Figure 2B. Response amplitudes are measured at a number of different log intensities for each wavelength, and sigmoidal curves are drawn through the points. The log intensities needed for any chosen criterion response can then be read from the sigmoidal curves by interpolation.

To shorten the experimental time, a simplification of this method is based on the 
Figure 2 - Schematic illustrations of flash and interferometric, linear-response methods. In $A-D$, the three points or curves represent the response amplitudes at three different wavelengths, $\lambda_{1}, \lambda_{2}$ and $\lambda_{3}$, with the criterion response amplitude elicited by three different intensities $I_{1}$, $I_{2}$ and $I_{3}$. In $A$, intensity is increased or decreased (as shown by arrows) until the response at each wavelength equals the criterion response amplitude. In B, complete V/ logl curves are measured at three different wavelengths, and sigmoidal curves are drawn through the points. The intensities needed at each of the wavelengths to elicit the criterion response are then determined by interpolation of the sigmoidal curves. Parallel curves indicate that the Principle of Univariance holds. In $D$, one complete $V /$ logl curve is measured and fitted with a sigmoidal curve. This sigmoidal curve is then slid along the intensity axis to coincide with response amplitudes measured at only one intensity at each wavelength. As in $B$, the intensity needed for a criterion response is found by interpolation. In the ramp method in $C$, the intensity starts at its lowest value at each wavelength and then is increased continuously until the amplitude of response reaches the criterion amplitude. Finally, the steps involved in the interferometric, linear-response method are shown in E. Both response and intensity are recorded during scanning of the interferometer, and the amplitudes versus time are converted by the Fast Fourier Transform to amplitudes versus wavelength. The intensities are corrected for the spectral sensitivity of the photomultiplier and are converted into quantum fluxes. Dividing the response by the intensity yields the spectral sensitivity, which may need to be corrected for the photoreceptor frequency response (since wavelengths are coded as frequencies in the interferogram).
A

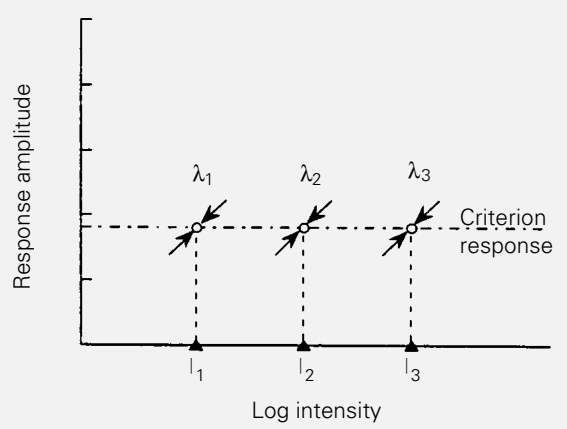

C

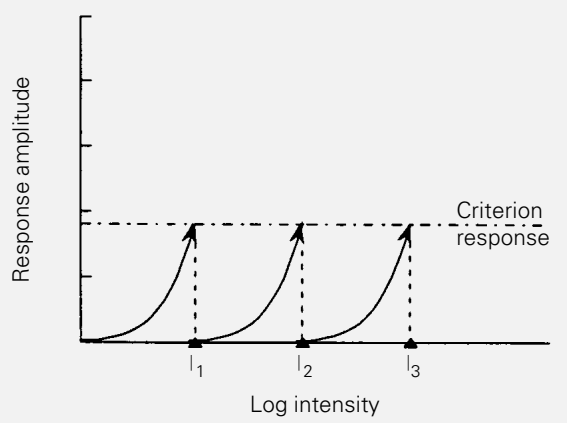

B

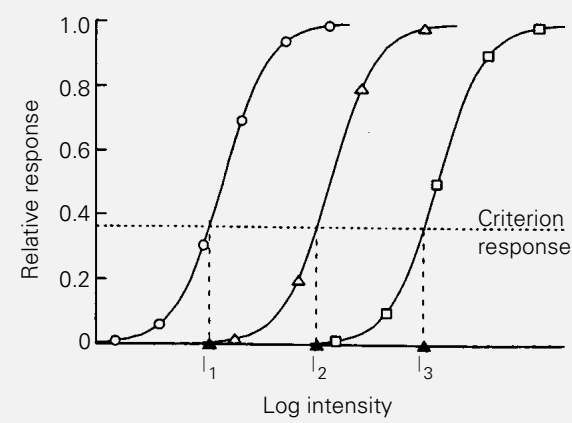

D

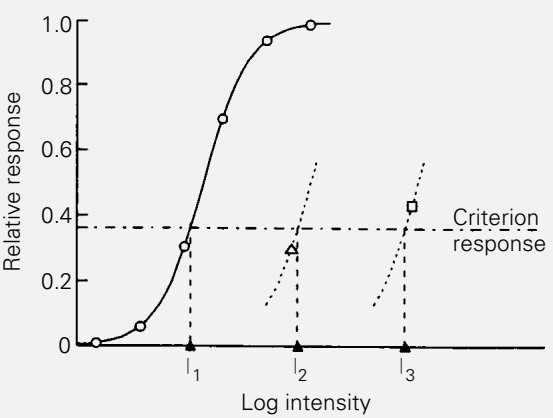

$\mathrm{E}$

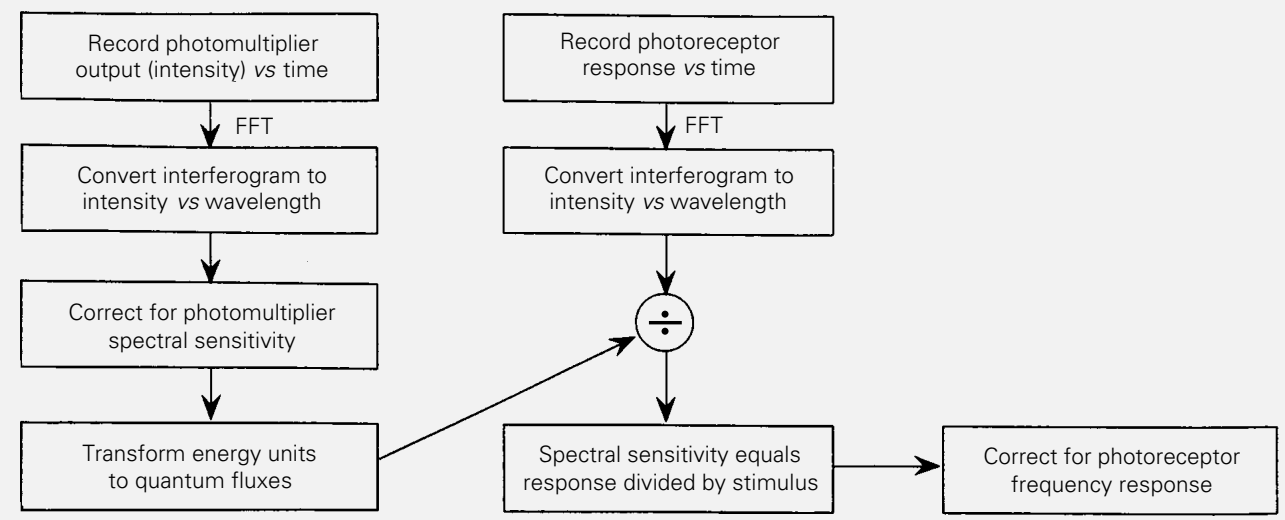


finding that $\mathrm{V} / \log$ I functions of most photoreceptors are parallel across the spectrum (as schematized in Figure 2B), consistent with the Principle of Univariance (1). Consequently, it can be sufficient to measure a V/ $\log$ I function at one monochromatic wavelength and the response to only one intensity at all other wavelengths. As schematized in Figure 2D, construction of the $S(\lambda)$ function in this way is done by interpolation from the response measured at each wavelength, using the V/logI curve to determine the intensity that would have been required for a criterion response amplitude at that wavelength. It is desirable to elicit nearly constant amplitudes of response by delivering approximately equal-brightness flashes at all wavelengths, thereby reducing the amount of interpolation needed from $V / \log$ f functions $(4,5)$. However, non-parallel V/logI functions have been found for small but not large response amplitudes for some photoreceptors in dragonfly median ocelli (6), and the sensitivities of these ocelli can change from a maximum in the green to a maximum in the UV with increasing levels of illumination, a reverse Purkinje shift (7). These cells clearly do not follow the Principle of Univariance.

The flash methods cited above present three drawbacks: first, for conventional measurements from dark-adapted eyes, data collection is lengthy due to the need to dark adapt the receptor between successive flash presentations. Second, the dark-adapted responses of photoreceptors to light flashes of constant intensity and duration can vary considerably from one flash to another. This often results in $S(\lambda)$ functions with double and triple peaks, which earlier were sometimes erroneously attributed to electrical coupling between photoreceptors or to the existence of more than one pigment in a photoreceptor cell (8-11). In addition, in dark-adapted cells there can sometimes be adaptational or facilitatory effects of one flash upon the response to a following flash so that even a train of equal intensity flashes of one wavelength may not elicit equal amplitude responses $(12,13)$. Third, results for a cell are not available immediately since they depend on calculations that cannot be done on-line.

A number of variations of the flash method have been developed to avoid some of the above drawbacks. For example, to determine spectral sensitivities of light-adapted UV receptors in flies, Hardie and Kirschfeld (14) scanned wavelengths with continuous, low intensity light and then scanned intensity ranges in discrete steps with a continuous light. Off-line, they digitized the continuous voltage responses with a graphic tablet and averaged 5 to 40 such scans of each type. Then, using the V/logI curve elicited by the discrete steps of light, they were able to determine the spectral sensitivities as in the flash methods described above, from the voltages measured during the wavelength scans.

Another variation, in which results are immediately available but which does not rely on square-shaped flashes, is the ramp method (15), shown in the flow chart of Figure 2C. At each wavelength, a shutter opens and a stepper motor-driven wedge rapidly increases the intensity ( $1 \log$ unit in $65 \mathrm{~ms}$ ) until the recorded response reaches a criterion amplitude, as detected by an analog comparator. The shutter then closes, a modified pocket calculator (HP 25) records the wavelength and wedge setting, computes the quantum flux from prestored calibrations, and outputs the wavelength and computed sensitivity to an X-Y plotter or storage oscilloscope. The wedge is then reset to maximum density, and the wavelength advanced for the next measurement. A spectral sensitivity function takes about one minute to measure and shows none of the spurious peaks found with the flash method (11).

\section{Linear-response methods}

In electrophysiological measurements, 
there can sometimes be a linear relationship between stimulus intensities and response amplitudes for small modulations of the stimulus, particularly for flicker of a constant illumination $(16,17)$. In the linear range of response, the response magnitudes are then proportional to the number of quanta which are effectively absorbed. Thus, spectral sensitivities can be determined simply in terms of relative amplitudes of response at different wavelengths, since these amplitudes are inversely proportional to the intensities which would have been needed to obtain a constant amplitude of response.

The computerized Fourier interferometric method of Gemperlein and colleagues (18-23) appears to be the only application of the linear response method. In the interferometric method, schematized in Figure 2E, a continuously scanning Michelson interferometer generates stimulus and response interferograms in a range of wavelengths which are frequency coded. By means of the Fast Fourier Transform (FFT), the amplitudes as a function of frequency are resolved into amplitudes as functions of wavelength. After appropriate corrections, the spectral sensitivities are determined by dividing the response amplitudes by the stimulus amplitudes (22). Complete spectral sensitivities for 40 wavelengths in the range of 300 to 700 $\mathrm{nm}$ can be determined in 7 to $16 \mathrm{~s}$. One complication of this method is that response amplitudes within the frequency bands of the interferogram may depend not only upon wavelength but also upon the frequency response of the cell. The dependence of amplitudes on frequencies has proven small for the frequencies used $(4-8 \mathrm{~Hz})$ and the photoreceptors tested (21), but the method does require that the frequency responses be measured separately using, for example, sinusoidally modulated light. Nonlinearities in response, if any, show up at second and higher harmonics of the frequencies in the interferograms and can either be ignored or used for further system description (22).

\section{Constant-response methods}

Methods which measure $S(\lambda)$ during continuous illumination $(14,22)$ eliminate sensitivity variations introduced by changes in the adaptation state as is also the case for constant-response methods. In constant-response methods, illumination is also continuous and the light intensity at each wavelength is adjusted until the response (AC or DC) matches a criterion amplitude. Perhaps the simplest of such methods is the color substitution method used by Neitz and Jacobs (24). These authors interleaved test and reference flickering beams (with dark periods between the light flashes) and manually changed the setting of a neutral density wedge in the test beam until the averaged periodic responses (ERGs) were judged to be nearly zero. Spectral sensitivity data took 45 to 60 min to collect and, as in the flash methods, were analyzed off-line. Gribakin (25) developed a more rapid color substitution method for ERGs and intracellular recordings in which test and reference beams alternated without intervening dark periods, and in which wavelengths were scanned with a motor-driven monochromator. During the spectral scans, any AC responses at the flicker frequency were detected by a phase-sensitive amplifier and fed back to vary the current through the test lamp until the AC responses were minimized. The quantum fluxes from the monochromator, measured with a constant quantum sensitivity photometer $(1<600 \mathrm{~nm})$, and the monochromator wavelength (position) were recorded on-line with an $\mathrm{X}$-Y recorder in the form of an inverted spectral sensitivity curve. As with other AC methods (see below), this method is insensitive to drifts in resting potentials of cells. Its chief disadvantage is the difficulty of obtaining sufficiently clean switching between reference and test lights so that no spurious transients in responses are generated, a problem obviated by the intervening dark periods in the method of Neitz and Jacobs (24). 
Other AC constant-response methods have involved flicker of only the test beam. Padmos and Norren (26) chopped the light beam and amplified the fundamental of the recorded human ERG with a vector voltmeter and fed its output back to a neutral density wedge controlled by a servo motor. The maximum velocity of the wedge was adjustable to avoid feedback oscillations. Each wavelength was determined by an interference filter that was combined with neutral density filters to transmit a constant energy. The log spectral sensitivities, based on radiant energies in the psychophysical tradition, could then be plotted on-line with an X-Y recorder in terms of the wedge density (rotation) for minimal ERG versus wavelength (position of the filter wheel). For 19 interference filters, a spectral sensitivity took about 5 min to measure.

Smakman and Pijpker (27) used a similar chopped light beam but employed analog integration during light-on and light-off to filter the noise in their intracellular responses and to obtain a peak-to-peak voltage for feedback control of a neutral density wedge that was controlled by a servo motor. Servo stability was obtained by an adjustable digital integrator of the peak-to-peak signal and by proportional-derivative control of the servo motor. In their experiments, each wavelength was set by an interference filter that was combined with neutral density filters to transmit a constant quantum flux. On-line plots of wedge position $v s$ wavelength then gave the spectral sensitivities. The spectral sensitivity that they illustrated with 7 wavelengths required $140 \mathrm{~s}$ (but could have been measured more rapidly).

In addition to AC constant-response methods, DC constant-response methods have also been used. In these, the DC response itself is fed back to control the light intensity. The first worker to use the DC constantresponse method for measuring spectral sensitivities was Franceschini $(28,29)$, who used a hard-wired analog system. Feedback sta- bility for the servo motor that controlled a neutral density wedge was obtained with a settling time of about $0.1 \mathrm{~s}$. Although response data for 20 wavelengths (set by interference filters rotated into the light beam by a stepper motor) could be collected within as little as $20 \mathrm{~s}$, the quantum flux for each combination of wavelength and measured neutral density still had to be calibrated offline after the experiment.

Yamada and Yasui (30) used a white light source filtered by interference filters and a fast, piezoelectric photomodulator to control the intensity through proportionalintegral-derivative (PID) feedback, manually adjustable for stability. Because of the speed of the modulator, Yamada and Yasui were able to determine both AC and DC sensitivities by adding a triangular wave and a DC voltage to set the criterion response amplitude. The AC and DC intensities of the monochromatic light on the eye were monitored with a photocell whose output was corrected off-line to give quantum fluxes.

\section{Computer-based constant-response methods}

The widespread development of microcomputer-based laboratory interfaces over the last 10 years has provided another way to obtain rapid, on-line determinations of spectral sensitivities. In the apparatus of Djupsund et al. (31), designed to measure photoreceptor adaptation, the low pass filtered, digitized voltage response of a cell controlled the intensity of a $555 \mathrm{~nm}$, green LED (intensity was linear with LED current) through a PID transfer function calculated by a microcomputer. Although the measuring system was fast, the wavelength of the LED could not be changed and so the apparatus could not measure spectral sensitivities. A more recent modification that uses a xenon lamp and a fast, servo-controlled neutral density wedge to control intensity can be used to measure spectral sensitivities (32). 
Another computer-based method is the DC constant-response method of de Souza et al. (33) and Menzel et al. (11). In this singlebeam method, shown schematically in Figure 1 , a computer controls stepping motors that drive a monochromator and a neutral density wedge. The computer steps the spectrum from ultraviolet to red or vice-versa. After each wavelength change, the response abruptly changes, and the computer uses the sign of a comparator to step the wedge density up or down until the photoreceptor response crosses a constant criterion value. This method, like the ramp method (15), thus finds intensities which elicit response amplitudes which bracket a criterion value to within the resolution of the wedge; sophisticated servo methods to stably control response amplitudes are not necessary. Hundreds of $S(\lambda)$ functions have been obtained with this method in many insect species $(11,34)$ and more recently in turtle retinal cells (de Souza JM, Zana Y, DeVoe RD and Ventura DF, unpublished data).

The advantages of such computerized constant-response methods for intracellular recordings are: 1) on-line presentations of $S(\lambda)$, which are calculated at the end of each spectral scan from the wedge positions using prestored calibration corrections, 2) greater number of sampled points (101 wavelength values can be tested in $20 \mathrm{~s}$ as opposed to about 20 wavelength values in $5 \mathrm{~min}$ in the flash methods), 3) greater speed of data collection, and consequently the possibility of several on-line repetitions to confirm the data before losing the cell, and 4) maintenance of the cell's adaptation level, since illumination is both continuous and of nearly constant brightness.

However, DC constant-response methods cannot be applied to the investigation of $S(\lambda)$ in cells without sustained tonic responses, since these methods are based on maintaining the DC response at or around a criterion level throughout a spectral run. For such cells, AC constant-response methods must be used instead. Of the existing AC constant-response methods (24-27,30), only Gribakin's method provides on-line presentations of $S(\lambda)$, but is limited to wavelengths less than $600 \mathrm{~nm}$ (because of the rhodamine dye used in his constant quantum sensitivity photodetector). This has led us to develop a computerized, single-beam, AC constant-response method (35), which is also schematized in Figure 1. Flicker $(8-50 \mathrm{~Hz})$ is produced either by a shutter (100\% modulation) or by modulation of a xenon lamp current (40\% maximum modulation). The elicited receptor potential is periodic and varies in amplitude over the spectrum. The system is designed to measure the peak-to-peak response voltage (PPV) and to determine when it crosses a pre-set criterion value. Wavelengths are scanned from 300 to $700 \mathrm{~nm}$, or vice-versa, in 4-nm steps. At each wavelength the periodic response is digitized at 1000 samples/s and smoothed by averaging 16 consecutive samples; then the peak-topeak amplitude of the smoothed samples is found and compared to the criterion. If they are unequal, the wedge is stepped to a denser or a less dense position depending on the sign of the difference. After the criterion value has been crossed, the wedge position corresponding to the closest match is recorded. The method is applicable to any type of visual cell which has a response with a strong phasic component, or to mass responses such as the ERGs or visual evoked potentials (VEPs). In addition, it can be used to measure $S(\lambda)$ of spectrally opponent neurons (the spectral opponency must first be determined by flash methods, however, since the AC method is insensitive to phases of responses).

\section{Discussion}

Most rapid, computer-based methods of measuring spectral sensitivities have been AC or DC constant-response methods (the exception is Fourier interferometry based on 
linear responses; this method also requires measurements of cell frequency responses (22)). The choice of an AC or a DC method depends in part on whether the electrophysiological responses of interest have sustained tonic components, in which case either AC or DC methods may be used, or whether the responses have primarily phasic response components, in which case AC methods alone are satisfactory. Examples of tonic responses are receptor potentials of photoreceptors; phasic responses include ERGs and responses of higher-order retinal neurons, such as lamina monopolar cells in insects and amacrine cells in vertebrate retinas.

Baseline instabilities, which are frequent in intracellular recordings due to movement of the animal, muscle contractions or other unwanted events, affect the DC constantresponse method because they cause fluctuations which are falsely interpreted as sensitivity changes. The AC constant-response method has the advantage of being relatively independent of baseline shifts, since the criterion is a peak-to-peak amplitude rather than a voltage level. As a consequence, the AC constant-response method can produce stable $S(\lambda)$ curves even under some kinds of unstable recording conditions. On the other hand, spectral scanning with the DC constant-response method is quicker, since it is only necessary to reach the criterion before stepping to the next wavelength. In order to reach the criterion in the AC constant-response method, it is frequently necessary to measure the peak-to-peak response a number of times: the program measures peak-topeak amplitude at each successive wedge position, and this operation is repeated as many times as needed to reach the criterion. This makes it slower than the DC method. Both constant-response methods have the important advantage that the light adaptation of the cell is maintained nearly constant during the experiment, since keeping responses close to the criterion at all wavelengths implies stimuli of nearly constant brightness, assuming that the Principle of Univariance (1) is valid.

Finally, computer control of light sources and computer digitization of recorded electrophysiological responses mean that almost all aspects of stimulation and analysis can be handled in software; there is minimal need for special purpose electronics $(32,35)$. This can be an advantage because any later modifications that might need to be made in stimulus or in analysis procedures can often be made more easily in software than in hardware. Thus, computerized constant-response methods can often use "off-the-shelf" electronic components, reducing the problems of assembling the physical parts of a system to measure spectral sensitivities. Shifting much of the development to writing software can, of course, be a difficulty in itself, but even these difficulties can now be mitigated with current high-level programming tools such as LabView (National Instruments), SuperScope (GW Instruments), or Visual Basic (Microsoft). In essence, it is possible today to assemble a system to measure spectral sensitivities by developing the preparation and the necessary optics with motorized control of stimulus parameters, and then connecting them together into a system via a computerized laboratory interface.

\section{References}

1. Naka KI \& Rushton WAH (1966). An attempt to analyse colour reception by electrophysiology. Journal of Physiology, 185: 556-586

2. Chaffee EL \& Hampson A (1924). Effects of varying the wavelength of the stimulating light upon the electrical response of the retina. Journal of the Optical Society of America, 9: 1-25
3. Goldsmith T (1964). The visual system of insects. In: Rockstein M (Editor), The Physiology of Insects. Vol 1. Academic Press, New York, 397-462.

4. Graham CH \& Hartline HK (1935). The response of single visual sense cells to lights of different wave length. Journal of General Physiology, 18: 917-931.
5. DeVoe RD (1972). Dual sensitivities of cells in Wolf spider eyes at ultraviolet and visible wavelengths of light. Journal of General Physiology, 59: 247-269.

6. Chappell RL \& DeVoe RD (1975). Action spectra and chromatic mechanisms of cells in the median ocelli of dragonflies. Journal of General Physiology, 65: 399419. 
7. Stange G (1981). The ocellar component of flight equilibrium control in dragonflies. Journal of Comparative Physiology A, 141: 335-347.

8. Bennett RR, Tunstall J \& Horridge GA (1967). Spectral sensitivity of single retinula cells of the locust. Zeitschrift für Vergleichende Physiologie, 55: 195-206.

9. Shaw SR (1969). Interreceptor coupling in ommatidia of drone honeybee and locust compound eyes. Vision Research, 9: 9991029.

10. Menzel R (1979). Spectral sensitivity and color vision in invertebrates. In: Autrum $\mathrm{H}$ (Editor), Handbook of Sensory Physiology. Springer-Verlag, Berlin, Heidelberg, New York.

11. Menzel R, Ventura DF, Hertel H, deSouza JM \& Greggers U (1986). Spectral sensitivity of photoreceptors in insect compound eyes: Comparison of species and methods. Journal of Comparative Physiology A, 158: 165-177.

12. Hanani M \& Hillman P (1976). Adaptation and facilitation in the barnacle photoreceptor. Journal of Insect Physiology, 67: 235-249.

13. Ventura DF \& Puglia NM (1977). Sensitivity facilitation in the insect eye. Journal of Comparative Physiology A, 114: 34-49.

14. Hardie RC \& Kirschfeld K (1983). Ultraviolet sensitivity of fly photoreceptors R7 and R8: evidence for a sensitising function. Biophysics of Structure and Mechanism, 9: 171-180.

15. Menzel R, Erber J \& Greggers U (1978). On-line computation of photoreceptor spectral sensitivity - a low-cost solution with a programmable pocket calculator. Vision Research, 18: 879-882.

16. DeVoe RD (1963). Linear relations between stimulus amplitudes and amplitudes of retinal action potentials from the eye of the Wolf spider. Journal of General Physiology, 47: 13-32.

17. Gemperlein R \& McCann GD (1975). A study of the response properties of retinula cells of flies using nonlinear identification theory. Biological Cybernetics, 19: 147-158.

18. Gemperlein R, Parsche H \& Paul R (1977). Dynamic spectral analysis of visual cells by Fourier spectroscopy. Naturwissenschaften, 64: 275-276.
19. Gemperlein R (1982). The determination of spectral sensitivities by Fourier interferometric stimulation (FIS). In: Niemeyer G \& Huber C (Editors), Documenta Ophthalmologica Proceeding Series. Dr. W. Junk, The Hague.

20. Adamczyk R, Gemperlein R, Paul R \& Steiner A (1983). Objektive elektrophysiologische Bestimmung der skotopischen und photopischen spektralen Empfindlichkeiten des menschlichen Auges mit Hilfe fourierinterferometrischer Stimulation (FIS). Fortschritte der Ophthalmologie, 80: 488-491.

21. Gemperlein R, Adamczyk R, Paul R \& Steiner A (1983). Die Vorteile fourierinterferometrischer Stimulation (FIS) bei der Bestimmung spektraler Empfindlichkeiten des menschlichen Auges. Fortschritte der Ophthalmologie, 80: 492-495.

22. Paul R, Steiner A \& Gemperlein R (1986). Spectral sensitivity of Calliphora erythrocephala and other insect species studied with Fourier interferometric stimulation (FIS). Journal of Comparative Physiology A, 158: 669-680.

23. Steiner A, Paul R \& Gemperlein R (1987). Retinal receptor types in Aglais urticae and Pieris brassicae (Lepidoptera), revealed by analysis of the electroretinogram obtained with Fourier interferometric stimulation (FIS). Journal of Comparative Physiology A, 160: 247-258.

24. Neitz J \& Jacobs GH (1984). Electroretinogram measurements of cone spectral sensitivity in dichromatic monkeys. Journal of the Optical Society of America A, 12: $1175-1180$

25. Gribakin FG (1981). Automatic spectrosensitometry of photoreceptors in Lethrus (Coleoptera, Scarabaeidae). Journal of Comparative Physiology A, 142: 95102.

26. Padmos P \& Norren DV (1972). The vector voltmeter as a tool to measure electroretinogram spectral sensitivity and dark adaptation. Investigative Ophthalmology, 11: 783-788

27. Smakman JGJ \& Pijpker BA (1983). An analog-digital feedback system for measuring photoreceptor properties with an equal response method. Journal of Neuroscience Methods, 8: 365-373.
28. Franceschini $N$ (1979). Voltage clamp by light: rapid measurement of the spectral and polarization sensitivities of receptor cells. Investigative Ophthalmology and Visual Science, 18 (Suppl): 5 (Abstract).

29. Franceschini N (1984). Chromatic organization and sexual dimorphism of the fly retinal mosaic. In: Borsellino A \& Cervetto L (Editors), Photoreceptors. Plenum, New York, 319-350.

30. Yamada M \& Yasui S (1988). Measurement of DC and AC spectral sensitivities of retinal horizontal cells by "voltage clamp by light". Journal of Neuroscience Methods, 24: 65-72.

31. Djupsund K, Kouvalainen E, Järvilehto $M$ \& Weckstrom M (1993). A digital feedback controller application - a light response control system to reveal photoreceptor adaptation. IEEE Transactions on Biomedical Engineering, 40: 205-208.

32. Djupsund K, Kouvalainen $E$, Järvilehto $M$ \& Weckstrom M (1995). A digital feedback controller application for studying photoreceptor adaptation by 'voltage clamp by light'. Journal of Neuroscience Methods, 62: 29-36.

33. de Souza JM, Menzel R \& Ventura DF (1985). Improved spectral sensitivity measurements in bee photoreceptors made with the constant-response method. Brazilian Journal of Medical and Biological Research, 18: 633 (Abstract).

34. Peitsch D, Fietz A, Hertel H, de Souza J, Ventura DF \& Menzel R (1992). The spectral input systems of hymentopteran insects and their receptor-based color vision. Journal of Comparative Physiology A, 170: 23-40.

35. de Souza JM, DeVoe RD, Schoepps C \& Ventura DF (1996). An AC constant-response method for electrophysiological measurements of spectral sensitivity functions. Journal of Neuroscience Methods, 68: 203-210. 